\title{
A Review of Image Resizing Technology Based on Importance Index
}

\author{
Zijuan Zhang ${ }^{1}$ and Baosheng Kang ${ }^{2 *}$ \\ ${ }^{1,2}$ School of Information Science and Technology, Northwest University, \\ $X i^{\prime}$ an 710127, China \\ 1'bluesky1023@126.com, ${ }^{2}$ bskang@163.com
}

\begin{abstract}
This text discusses the feature of the existing methods relating to content-aware image resizing in detail, compares the performance and effect between different methods, and analyzes the merits and demerits about different algorithms. The basic process in contentaware image resizing are segmented into two steps: one of the steps is the identification of image content, which is generally computed by the product of image gradient and vision significance, and it reflects the different sensitivity that human eyes may feel towards different areas in image; Present articles regarding to content-aware image resizing mainly focus on the resizing technology that is the second step. According to the traits of different methods, this article divides the methods into three sorts: based on seam carving, image deformation and multi-operation.
\end{abstract}

Keywords: Resizing, Seam carving, Deformation, Multi-operation

\section{Introduction}

In the resizing step, the core of the content-aware image resizing method is that trying to keep main object area with the higher important degree in image invariant and changing the background area with lower degree in order to satisfy the target size. According to the traits in different methods, this article divides the methods into three sorts: based on seam carving, image deformation and multi-operation.

\section{The resizing technology based on seam carving}

The resizing algorithm based on seam carving that has been suggested by Shamir and Avidan[1] is also considered as backward method. The algorithm of seam carving is a image retargeting method by continuously inserting or removing seams which pass through the image totally. The seam's width is one pixel only, while removing the seam, the size of the image will be smaller, but if copying the seam, the measure of the image will be larger. Disposeing the horizontal(vertical) seam will alter the height(width) of the image. For keeping the dominating object of the image's content, the method of the seam carving inserts and removes the seam with the minimal energy at every turn. The seam energy is calculated by the sum of pixels' energy in each seam. In the paper [1], the pixel's importance degree is countted by the gradient. Let I be a size of $n^{*} m$ image and also regard a vertical seam as :

$$
S^{\nu}=\left\{S_{i}^{\nu}\right\}_{i=1}^{m}=\left\{(i, x(i)\}_{i=1}^{m}, \text { s.t. } \forall i,|x(i)-x(i-1)| \leq 1\right.
$$

* Corresponding author 
here $x(i)$ is a map: $x[1, \cdots, m] \rightarrow[1, \cdots, n]$. That is to say, a vertical seam is regarded as an 8-connected route constituted by pixels from top to bottom in the image, including one, and merely one pixel in image's every row. Analogously, a horizontal seam is also regarded as:

$$
S^{h}=\left\{S_{j}^{h}\right\}_{j=1}^{n}=\left\{(y(j), j\}_{j=1}^{n}, \text { s.t. } \forall j,|y(j)-y(j-1)| \leq 1\right.
$$

here $y(j)$ is a map : $y[1, \cdots, n] \rightarrow[1, \cdots, m]$. In a similar way, that is to say, a horizontal seam is regarded as an 8-connected route constituted by pixels from left to right in the image, including one, and merely one pixel in image's each column.

Preset an energy function $e$, we could define a seam's cost of as: $E(s)=\sum_{i=1}^{m} e(i, x(i))$, We find the the optimal seam $s^{*}$ that minimizes the cost of this seam:

$$
S^{*}=\min _{s} E(s)=\min _{s} \sum_{i=1}^{m} e(i, x(i))
$$

In the paper [1], the optimal seam can be seeked out by using dynamic programming algorithm. At first, it is to scan the image's pixels from the second row to the last row and count the cumulative energy with minimum $M$. With regard to all possible copular seams for each pixel item $(i, j)$ :

$$
M(i, j)=e(i, j)+\min (M(i-1, j-1), M(i, j-1), M(i+1, j-1))
$$

In the references $[3,8,9,10,11]$, the Backward algorithm is further improvement. In our experiment way, the Backward algorithm is improved through added some energy. We set new norm because the original method selects to delete the seam of the least amount of content energy in the image, overlooking content energy that is added into the resized picture.

The paper [3] presents new method based on seam carving, this method increases the energy item that is created by new neighborhood that is appeared after removing pixels. In the Figure 1, if removing vertical seam, it may produce three new types of neighboring pixels.

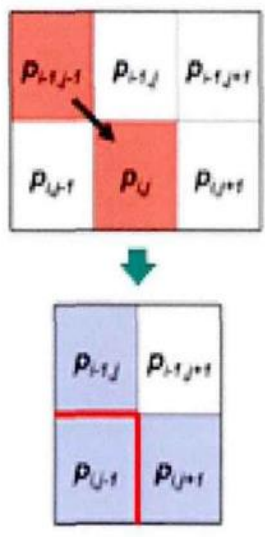

(a)

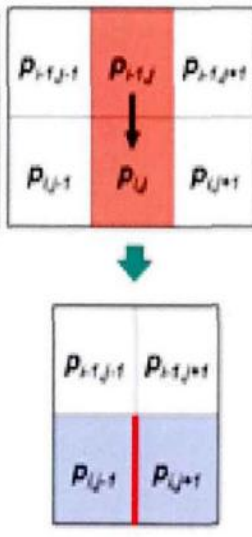

(b)

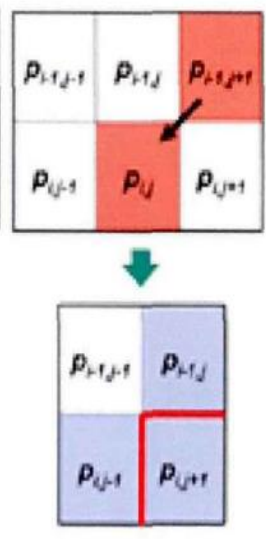

(c)

\section{Figure 1. New Types of Content Energy( Red Part Marked by Bold Lines)}

For each possible cases in above three types, we can define the cost of the three types $C_{L}(i, j) 、 C_{U}(i, j)$, and $C_{R}(i, j)$ respectively: 


$$
\begin{aligned}
& \text { (a) } C_{L}(i, j)=|I(i, j+1)-I(i, j-1)|+|I(i-1, j)-I(i, j-1)| \\
& \text { (b) } C_{U}(i, j)=|I(i, j+1)-I(i, j-1)|+|I(i-1, j+1)-I(i-1, j-1)| \\
& \text { (c) } C_{R}(i, j)=|I(i, j+1)-I(i, j-1)|+|I(i-1, j)-I(i, j+1)|
\end{aligned}
$$

In above formula, $I(i, j)$ is the pixel located in $(i, j)$ 'color value. So, with respect to vertical seam, each cost defined by $M(i, j)$ is updated applying the following norm:

$$
M(i, j)=e(i, j)+\min \left\{\begin{array}{c}
M(i-1, j-1)+C_{L}(i, j) \\
M(i, j-1)+C_{U}(i, j) \\
M(i+1, j-1)+C_{R}(i, j)
\end{array}\right.
$$

Literature[8] proposed the algorithm relating to succession seam carving. This algorithm always finds the seams number that exceed the amount which should deletes or copy, and lets the seams bear the certain proportion of removing(copying) amount, that is density of resample. In this way, the removing(copying) amount of a seam is in proportion to its energy. The literature[9] can remove the more than 1 pixel width and carry out the function which shift the main object, so this algorithm is also be called Shift-map method. The literature[10] considers that removing and copping the neighboring seams simultaneously may destroy the total vision effect. So when removing the seam, it add the removing seam energy to neighboring pixels, this way can memory the removing pixels information a certain extent. The literature[11] achieve the aim that keep the image feature by means of adjusting the seams which pass the feature points in image. In addition, The literature[12-19] also improve the seam carving technology.

Among all the seam carving algorithms, the Backward algorithm's status is undisputed, its creativity and innovation are superior to other methods.

\section{The Image Retarget based on Deformation}

The resizing based on image deformation [2, 4-7, 20-27] is another effective technology relating to content-aware image process. At first, this method divides the image into grids, then with the restraint of target image size, keep the main body grids with high important degree invariant(or change these grids with equal ratio) and let the unimportant background with low importance deformed, then produce the new deformation grids, ultimately, with the backing mapping of corresponding grids produces the target image.

At the Siggraph Asia conference in 2008, Wang [7] putted forward a Optimized scale-andstretch picture retargeting algorithm(SNS). Firstly, this algorithm divided the picture into many grids $(V, E, F), V$ is the set fo vertexes, $E$ is a set of borders, and $F$ is the set fo grids. $V=\left[v_{0}^{T}, v_{1}^{T}, \cdots v_{\text {end }}^{T}\right]$ and $v_{i} \in R^{2}$ manifest the initial position about vertexes. For resizing the size of $m \times n$ image to the arbitrary measure of $m^{\prime} \times n^{\prime}$, we can fix the corner vertex $v_{0}$ in top left and have the user appoint the new place to corner vertex $v_{\text {end }}$ in the below right. Other vertexes in borders then slide along borders under the rectangle shape's constraint. We have found the question about deflection grids $V^{\prime}=\left[v_{0}^{\prime T}, v_{1}^{\prime T} \cdots v_{\text {end }}^{\prime T}\right]$. For every $f \in F$ 's deflection volume, SNS way counts the distance between deflection mesh $v^{\prime}$ 
and original mesh $v$ 's unified scale version $s_{f} v+t$ ( $s_{f}$ is the retargeting factor, t can be seen the translation volume), for measuring the grid's deflection $D_{u}(f)$ :

$$
D_{u}(f)=\sum_{\{i, j\} \in E(f)}\left\|\left(v_{i}^{\prime}-v_{j}^{\prime}\right)-s_{f}\left(v_{i}-v_{j}\right)\right\|^{2}
$$

In the end, every grid deflection's effect about total grids' deflection $D_{u}$ is closely related to corresponding grid's content of important degree $w_{f} . w_{f}$ is counted by the average of grid pixels' energy. Hence, the total deflection volume $D_{u}$ is $D_{u}=\sum w_{f} D_{u}(f)$.

According to the fact that most main objects in the image ưstrally take up the continuous grids, SNS norm keeps the main object's character by minimizing the mesh lines' curling energy. The method is maintaining the border orient and adjusting its length. Meanwhile, SNS algorithm defines the mesh line curling energy $D_{l}$ as:

$$
D_{l}=\sum_{\{i, j\} \in E}\left\|\left(v_{i}^{\prime}-v_{j}^{\prime}\right)-l_{i, j}\left(v_{i}-v_{j}\right)\right\|^{2}, \quad l_{i, j}=\left\|v_{i}^{\prime}-v_{j}^{\prime}\right\| /\left\|v_{i}-v_{j}\right\|
$$

At the last, by minimizing the sum of grid's deflection deflection volume, gird lines curling energy $D$ can be seen as:

$$
D=D_{u}+D_{l}
$$

In the process of calculation, at first, SNS norm supposes the grid vertex as $v^{\prime}$, counts optimally $s_{f}$ of the grid's retargeting parameter, then under the limitation of the resized image border updates the mesh vertex $v^{\prime}$, at the end achieves iteratively.

If you want to divide the picture into grids by applying the SNS algorithm, it is easy to introduce the special grid that have not only the main body pixels but also background pixels, can not realize the coincidence of border vertexes and image object feature, and isn't beneficial to the maintenance of local trait. To this situation, the literature[23] identify the border feature point through the main body object, connect the grid of feature points with feature points in order to produce the different controlling points, at last keep the image feature through optimizing controlling points. The literature [24] improved the optimizing function in literature [23] in order to avoid the deformation and rotating in important area.

The literature $[2,22]$ are similar to SNS method, and also divide the image into grids. When describing the scaling ratio about grids, it is not similar with SNS method which apply the agreed resizing factor $s_{f}$, but distinguish the resizing factor $a_{i, j}^{1}, a_{i, j}^{2}$ regarded to x,y axis. Then according to important degree in grid, adjusting $r=a_{i, j}^{1} / a_{i, j}^{2}$ the degree of closeness to 1 in order to control the corresponding grid's deformation volume. For higher important degree grid, we try to adjust the $r$ equal to 1 . But for lower degree grid, we permit the $r$ depart from the 1 in order to fit the target image's size.

The algorithm and grid energy in literature [2, 22]is similar, the main distinction between them is the solution to optimize the grid energy. The literature optimizes the single grid energy partly in iteration to solute the resizing factor $a_{i, j}^{1}, a_{i, j}^{2}$, then solve the grid points' position through the total optimization towards to the sum of all the grids, this way is called as LG(local-Global) method. But the literature [22] apply the convex quadratic program method, this way is called as QP methos.

In effect, SNS way [7] achieve better results that keep the main body's structure in image through the strategy of maintaining the scaling factor equally. But this method doesn't 
consider that different factors may produce various effect to grids, so the main body in result image resizes along with the original image's resizing, for example the beerhouse (Figure 2) in result image resizes much smaller but imbalance to other buildings' ratio, and affects the total vision effect. Because the QP method apply the local-total optimizing strategy to get result, the result cannot achieve the total optimal when achieving the local optimal, and the result's effect is instability. For instance the beerhouse is stretched much more and not keep the original ratio(Figure 2). QP method [22] apply the convex quadratic domestication to solve the total resolution and achieve the better result (Figure 2) through adding the energy of forbidding the grids fold and amplifying the important area.

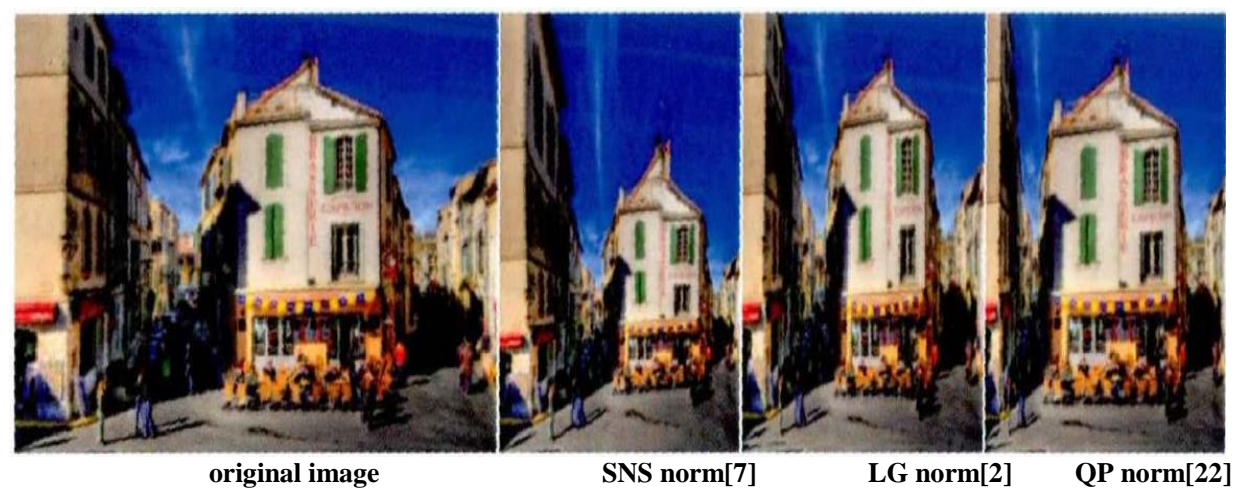

Figure 2. The Resizing Effect based on Image Deformation Quantity(Paper[22])

\section{Multi-operation Resizing}

Multi-operation method [28-31, 33] is a combination of seam carving, traditional scaling and cropping or only the first two way's combination in order to achieve the content-aware image resizing. The key point in this way is how to decide the operations' amount and order. The direct idea is that lists all the order and amount results, then choose the optimal result. If permitting reduce and amplification be cross, the order of operations is infinite. So when reducing(amplification) the image, we should firstly set every operation only reducing(amplification) process. Under this premise, if applying $\mathrm{m}$ operations to change the image size w, the operation order is $m^{v}$, it is also big, so present multi-operation ways usually give some additional assumption to reduce computation. Frequently-used premises has been divided into two types: one is finding regular path [28], according to the fact that the operation's amount's effect is bigger than the order, fixing the operation order may reduce to find the optimal operation amount; The other is finding the mix path[28], firstly fixing every sub-operation's amount $k(k \geq 1)$, then deciding the operation order's length $\mathrm{n}$, finally applying dynamic programming to decide every operation's type. Additionally, when we finding the optimal operation type, we only retain the optimal value towards to every order that has same operation amount but different order in order to reduce the computation. If there has two type operation's multi-process, the way to finding the path shows in Figure 3: 


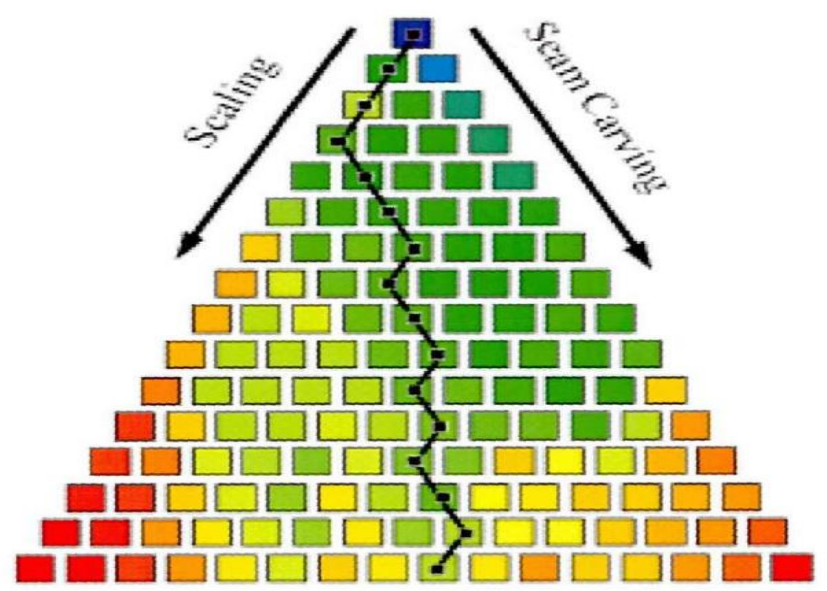

Figure 3. Applying Dynamic Programming Algorithm to Look for the Optimal Mixed Path that only Employ Two Single Operations [28]

After deciding the way to finding operations' order and amount, the key point to this method is how to design a good measure criterion to weight similarity between the original image with results, this is the main difference among all the multi-operations.

At the SIGGRAPH in 2009, Rubinstein proposed a multi-operation way [28]. The article pointed out that multi-operation's result is better than single operation, because this text through statistical analysis towards user's satisfied degree to results that is operated by various ways (single seam-carving, traditional scaling, or the combination of three methods).

Rubinstein's multi-operation [28] apply Bi-Directional Warping(BDW) to measure the difference between the target image with original. BDW contains the difference between target image with original (reflecting the coupling with original and target, that is how much noise in target image that is taken by original) and the difference between the original image with target (reflecting the integrity that target towards original image information). At first, BDW divides the original image into blocks, then finding the most similar block in original image that is applied by dynamic programming. The finding of optimal matching block follows the consistence between the matching block with original's order, and only permitting some to one, not one to some. Ultimately, The average of computing all the blocks' pixels color value is regarded as the difference between the images (see Figure 4). According to the difference value, the literature [28] apply the regular path and mixed path respectively, find the optimal operation order and get the corresponding result.

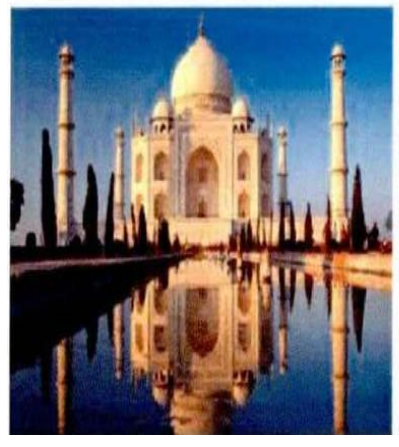

original image(S)

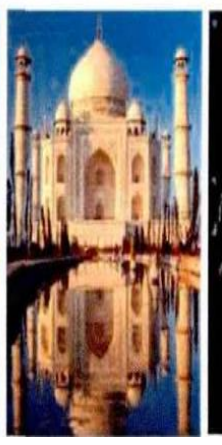

target image

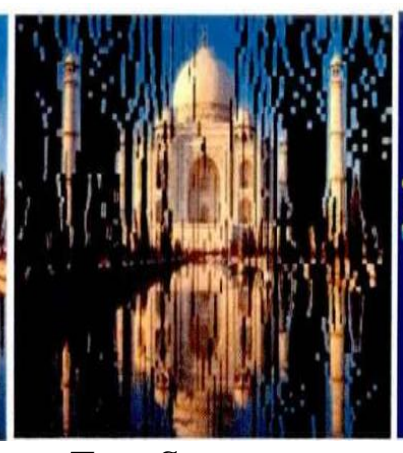

$T \rightarrow S$ matching blocks $(8 * 8)$

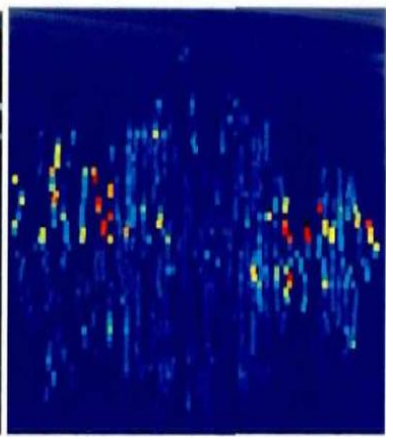

$L 1$ distance between blocks

Figure 4. Using BDW to Find the Optimal Matching Blocks[28] 


\section{Conclusion}

In conclusion, up to now there is no objective or subjective precise standard that evaluate the different resizing methods' performance[32]. Hence, this text give some qualitative analysis towards to three resizing technologies' feature. At first, seam carving and deformation algorithm are more speeder than the multi-operation, especially multi-operation based on block-match. Secondly, from effect views, every method has its advantage and fitting image, there is no way that is universal to any image.

The features of the seam carving come from the operation belongs to pixels. Because of the pixels, the results are precise; The operation is discrete, so the result's color is most near to original image. But the operation is discrete, so the result' structure information is easy to destroyed, and produced manual noise. Hence, seam carving fits the image that has smooth background or less structure, in addition, seam carving method can also delete the object.

The essence of the multi-operation is that balance the main body's deformation, structure's destroying, manual noise and image content's loss, and then compromise. Usually, the more ideal condition is that seam carving towards image first, when the seam carving destroy the image structure's information, produce more manual artificial noise or delete the background information that affect the total vision effect, then give up the seam carving and estrange scale uniformly. In this way, scale's continuity may keep the image's ratio, and control the manual noise. But, most operation in optimizing process can not find the most optimal result, and only produce better result. Hence, people have the feeling that is not most perfect but better result towards to multi-operation

The image deformation's resizing, which is restrained by important degree and border and has scale's continuity and keep the feature of image's main body, can give resizing factor belonging to different grid though optimizing the sum energy of grids. But when the grid degenerates to a point or a line, the super compression of corresponding area may produce discontinuity in image content.

\section{Acknowledgements}

The authors are grateful to the anonymous referees for their valuable comments and suggestions to improve the presentation of this paper.

\section{References}

[1] S. Avidan and A. J. Shamir, ACM Transactions on Graphics, vol. 3, no. 26, (2007).

[2] Z. Karni, D. Freedman and C. Gotsman, J. In Computer Graphics Forum, vol. 5, no. 28, (2009).

[3] M. Rubinstein, A. Shamir and S. Avidan, ACM Transactions on Graphics, vol. 3, no. 27, (2008).

[4] L. Feng and M. Gleicher, "Automatic Image Retargeting with Fisheye-View Warping", Proceedings of the 18th Annual ACM Symposium on UIST, New York, USA, (2005).

[5] J. Shi, Y. Guo and Z. Du, Journal of software, zk, vol. 19, (2008).

[6] Y. Guo, F. Liu and J. Shi, IEEE Transactions on Multimedia, vol. 5, no. 11, (2009).

[7] Y.-S. Wang, C.-L. Tai, O. Sorking and T.-Y. Lee, ACM Transactions on Graphics, vol. 5, no. 28, (2009).

[8] L. Zhi, Y. Hongbo and S. Liquan, Optical Engineering, U.S.A., vol. 1, no. 49, (2010).

[9] Y. Pritch, E. Kav-Venaki and S. Peleg, "Shift-Map Image Editing", Proceedings of the Twelfth IEEE ICCV, Florida, USA, (2009).

[10] C. Sunghyun, C. Hanul and Y. Matsushita, "Image Retargeting Using Importance Diffusion", Proceedings of the ICIP, Florida, USA, (2009).

[11] P. Deng, Degree paper, Zhejiang University Press, Hangzhou, (2008).

[12] N. Hill and P. Eslambolchilar, "Seam carving for enhancing image usability on mobiles", Proceedings of the 22nd British HCI Group Annual Conference on HCI, Liverpool John Moores University, UK, (2008).

[13] R. Nakashima, K. Utsugi and K. Takahashi, J. IEICE Transactions, D(6), 94, (2011).

[14] J.-W. Han, K.-S. Choi and T.-S. Wang, "Wavelet based seam carving for content-aware image resizing", Proceedings of the International Conference on Image Processing, Cairo, Egypt, (2009). 
[15] J. Chen, L. Miao and X. Liu, World Wide Web, vol. 3, no. 14, (2010).

[16] A. Mansfield, V. Peter and Gehler, "Scene Carving: Scene Consistent Image Retargeting", Proceedings of the 11th European Conference on Computer Vision, Heraklion, Crete, Greece, (2010).

[17] C. Sunghyun, C. Hanul and Y. Matsushita, "Image Retargeting Using Importance Diffusion", Proceedings of the ICIP, Florida, USA, (2009).

[18] J. Scott, R. L. Tutwiler and M. Pusateri, "Hyper-spectral content aware resizing", Proceedings of the 37th IEEE Applied Imagery Pattern Recognition Workshop, Washington, DC, USA, (2008).

[19] A. Srivastava and K. Kishore Biswas, "Fast Content Aware Image Retargeting", Proceedings of the Sixth Indian Conference on Computer Vision, Graphics \& Image, Processing Bhubaneswar, India, (2008).

[20] T. Ren, H. Liu and G. J. Wu, Journal of software, vol. 0, no. 21, (2010).

[21] R. Gal, O. Sorkine and D. Cohen-Or, "Feature aware texturing. Proceedings of the Eurographics Symposium on Rendering", Goslar, Germany: Eurographics Association, (2006).

[22] C. Renjie, D. Freedman and Z. Karni, "Content-Aware Image Resizing by Quadratic Programming", Proceedings of the 2010 IEEE CVPR WorkShop on NORDIA, Florida, USA, (2010).

[23] G.-X. Zhang, M.-M. Cheng and S.-M. Hu, "Computer Graphics Forum Special issue of Pacific Graphics", vol. 7 , no. $28,(2009)$.

[24] L. Lei, Journal of computers, vol. 10, no. 33, (2010).

[25] H. Bao and X. Li, "Non-uniform Mesh Warping for Content-Aware Image Retargeting", Proceedings of the 8th International Conference on Image Analysis and Recognition, Burnaby, BC, Canada, (2011).

[26] P.-Y. Laffont, J. Y. Jun and C. Wolf, "Interactive content-aware zooming", Proceedings of the Graphics Interface 2010 Conference, Ottawa, Ontario, Canada, (2010).

[27] S.-F. Wang and S.-H. Lai, "Fast structure-preserving image retargeting", Proceedings of the IEEE International Conference on Acoustics, Speech, and Signal Processing, Montreal, Canada, (2009).

[28] M. Rubinstein, A. Shamir and S. J. Avidan, ACM Transactions on Graphics, vol. 3, no. 28, (2009).

[29] W.-M. Dong and J.-C. Paul, “Adaptive content aware image resizing”, LIAMA, Tech. Rep., (2009).

[30] W.-M. Dong, N. Zhou and J.-C. Paul, ACM Transactions on Graphics, vol. 5, no. 28, (2009).

[31] W.-M. Dong, X.-P. Zhang and N. Zhou, "Fast multi-operator image resizing", LIAMA,Tech. Rep., (2009).

[32] M. Shi, Degree paper, Yunnan University Press, Kunming, (2011).

[33] D. Simakov, Y. Caspi and E. Shechtman, "Summarizing visual data using bidirectional similarity", Proceedings of the IEEE Conference on CVPR, Florida, USA, (2008).

\section{Authors}

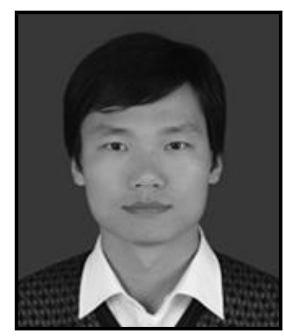

Zijuan Zhang, he received his B.S. degree in electronic engineering from Xi'an University of Posts and Telecommunications, China in June 2005 and his M.S. degree in mathematics from Shaanxi Normal University, China in June 2008. He is currently working towards his D.E. degree in Computer software and theory at Northwest University, China. His current research interest includes image and Graphics processing.

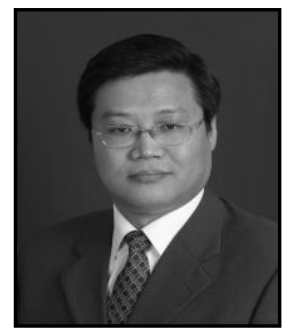

BaoSheng Kang, he received his B.S. degree and M.S. degree in mathematics from Northwest University, China in 1982 and 1985. Then he received his D.E. degree in Computer science from Northwestern Polytechnical University in 1991. His current research interest includes image processing, image retrieval and Computer Graphics. 